\title{
EVALUACIÓN DE LAS MANIFESTACIONES NEUROLÓGICAS EN PACIENTES DIAGNOSTICADOS CON COVID-19. UNA REVISIÓN SISTEMÁTICA
}

\section{Evaluation of neurological manifestations in patients diagnosed 'with COVID-19. A Systematic Review}

\author{
Juan Menaa ${ }^{a}$, Soraya Peñab, Saul Polancoc, Jorge Rosario ${ }^{d}$, Sebastián Ureña ${ }^{e}$, \\ Francisco Villegas ${ }^{f}$, Camila Yabrag, Jhonatan Rojas', Priscila Hernándezi y Paulette Reyes ${ }^{j}$
}

Recibido: 12 de abril, 2021 • Aprobado: 16 de junio, 2021

Cómo citar: Mena J, Peña S, Polanco S, Rosario J, Ureña S, Villegas F, Yabra C, Rojas J, Hernández P, Reyes P. Evaluación de las manifestaciones neurológicas en pacientes diagnosticados con COVID-19. Una revisión sistemática. cysa [Internet]. 12 de octubre de 2021 [citado 15 de octubre de 2021];5(3):69-0. Disponible en: https://revistas.intec.edu.do/index.php/cisa/article/view/2306

\section{Resumen}

Introducción: la enfermedad coronavirus 2019 (COVID19), causada por el virus llamado SARS-Cov-2, fue declarada pandemia por la Organización Mundial de la Salud (OMS). Afecta principalmente a los sistemas respiratorio y cardíaco, influyendo en otros como el sistema nervioso. En este sentido, la pregunta de investigación que orienta este estudio es: ¿̨cuáles serían las manifestaciones neurológicas en adultos diagnosticados con COVID-19?

Metodología: se realizó una revisión sistemática a través de los metabuscadores: PubMed, American Academy of Neurology (AAN), SciELO, ELSEVIER, y, finalmente, MedScape. Los criterios de inclusión fueron: investigaciones científicas sobre COVID-19 publicadas durante el periodo 2019, investigaciones en español y/o inglés, e

\footnotetext{
a Neurólogo. Instituto Tecnológico de Santo Domingo (INTEC). Correo-e: juan.mena@intec.edu.do ORCID: 0000-0002-0542-373

b Estudiantes de Medicina del Instituto Tecnológico de Santo Domingo (INTEC). ORCID: 0000-0002-7308-903X Correo-e: 1094964@est. intec.edu.do

c Estudiantes de Medicina, (INTEC). ORCID: 0000-0002-06474982 Correo-e: 1095028@est.intec.edu.do

d Estudiantes de Medicina, (INTEC). ORCID: 0000-0001-8267476X Correo-e: 1087971@est.intec.edu.do

e Estudiantes de Medicina, (INTEC). ORCID: 0000-0003-36617574 Correo-e:1094273@est.intec.edu.do
}

\begin{abstract}
Introduction: The coronavirus disease 2019 (COVID-19), caused by the virus called SARS-Cov-2 was declared a pandemic by the World Health Organization (WHO). It mainly affects the respiratory and cardiac system, influencing other systems such as the nervous system. The research question is: What would be the neurological manifestations in adults diagnosed with COVID-19?
\end{abstract}

Methodology: Systematic review, in which the metasearch engines were used: PubMed, American Academy of Neurology (AAN), SciELO, ELSEVIER, and finally, MedScape. The inclusion criteria were: scientific research on COVID-19 published during the 2019 period, research in Spanish and/or English, research with a focus on neurological manifestations; the ROBVIS tool was

\footnotetext{
f Estudiantes de Medicina, (INTEC). ORCID: 0000-0002-15367716 Correo-e: 1095233@est.intec.edu.do

g Estudiantes de Medicina, (INTEC). ORCID: 0000-0002-17791529 Correo-e:1093073@est.intec.edu.do

h Estudiantes de Medicina, (INTEC). ORCID: 0000-0001-80708145 Correo-e: 1069562@est.intec.edu.do

i Estudiantes de Medicina, (INTEC). ORCID: 0000-0001-67768442 Correo-e: 1061212@est.intec.edu.do

j Estudiantes de Medicina, (INTEC). ORCID: 0000-0003-41210074 Correo-e: 1070461@est.intec.edu.do
} 
investigaciones con enfoque en manifestaciones neurológicas; para el manejo del sesgo se utilizó la herramienta del ROBVIS y la revisión se realizó bajo la guía PRISMA.

Resultados: se seleccionaron un total de 32 artículos, 14 revisiones sistemáticas y 18 estudios observacionales (series de casos y reportes de caso). Las manifestaciones neurológicas en pacientes diagnosticados con COVID19, obtenidas de 30 artículos, fueron: cefaleas, mialgias, anosmia, ageusia. El grupo etario entre 50 y 70 años fue el más predominante al presentar manifestaciones neurológicas por COVID-19, disponiendo de un total de 14 artículos. Las condiciones neurológicas preexistentes más predominantes son el Alzheimer y el Parkinson, extraídas de un total de 11 artículos.

Conclusión: no hay duda de que la COVID-19 es sumamente variable en cuanto a su forma de presentación, al punto de tener una notable incidencia y variabilidad de efectos sobre el sistema nervioso. Afectando en mayor medida a personas de edad avanzada y con condiciones neurológicas de base.

Palabras clave: COVID-19; manifestación neurológica; SARS-CoV-; complicación neurológica, sistema nervioso central; sistema nervioso periférico.

\section{Introducción}

El síndrome respiratorio agudo grave coronavirus 2 (SARS-CoV-2, por sus siglas en inglés) causante de la enfermedad coronavirus 2019 (COVID-19) traza sus orígenes en China, Wuhan, y fue declarada pandemia el 26 de marzo del 2020 por la Organización Mundial de la Salud $(\mathrm{OMS})^{1}$. Esta enfermedad, en ocasiones asintomática, puede presentar síntomas tales como: fiebre, tos seca, fatiga, mialgia, disnea, anosmia y ageusia, pero en casos más severos complicaciones como: neumonía, síndrome respiratorio agudo grave (SARS) y problemas cardíacos ${ }^{1,2,3,4}$. Según varios estudios, el virus presenta manifestaciones neurológicas, las cuales pueden manifestarse de una forma leve (mareos, ageusia, anosmia y cefalea) moderada (mialgia, ataxia y parestesia) o used to manage bias. The review was conducted under PRISMA guidance.

Results: A total of 32 articles, 14 systematic reviews and 18 observational studies (case series and case reports) were selected. Neurological manifestations in patients diagnosed with COVID-19 were headache, myalgia, anosmia, ageusia obtained from 30 articles. The age group between 50 and 70 years was the most predominant when presenting neurological manifestations by COVID-19, having a total of 14 articles. The most predominant pre-existing neurological conditions are Alzheimer's and Parkinson's, extracted from a total of 11 articles. Conclusion: There is no doubt that COVID-19 is highly variable in its form of presentation, to the point of having a notable incidence and variability of effects on the nervous system. Affecting to a greater extent people of advanced age and with a preexisting neurological condition.

Keywords: COVID-19; neurological manifestation; SARS-CoV-2; neurological complication; central nervous system; peripheral nervous system.

grave (encefalopatía, enfermedades cerebrovasculares, encefalitis e hipoxia cerebral $)^{5,6,7}$.

La relación existente entre la COVID-19 y la presentación de manifestaciones neurológicas fue demostrada por primera vez en una revisión realizada en tres hospitales de Wuhan, China, con un total de 214 pacientes, en el cual un $25 \%$ presentó síntomas relacionados a disfunciones del SNC, donde se incluyen $17 \%$ mareos, $13 \%$ cefalea, $7.5 \%$ alteración de la consciencia, un $3 \%$ presentó enfermedades cerebrovasculares agudas, $0.5 \%$ presentó ataxia, y un $0.5 \%$ convulsiones ${ }^{5}$. Asimismo, en un grupo de 58 pacientes cuya edad media fue 63 ańos, se muestran complicaciones neurológicas en un $84 \%$, constituidas por agitación, confusión, signos del tracto corticoespinal (67\%), síndrome disejecutivo 
al momento del alta en pacientes con signos corticoespinales previos en un $36 \% .^{4}$.

El virus entra al sistema nervioso central (SNC) por invasión directa al tejido neural y mediante una respuesta inflamatoria ${ }^{1,4}$. El mecanismo de invasión se realiza a través de la unión al receptor de la enzima convertidora de la angiotensina II (ECA 2) en las células gliales del cerebro y neuronas espinales ${ }^{4,8}$. Este invade el sistema nervioso central (SNC) poco después de la infección, accede al líquido cefalorraquídeo y a las subregiones del cerebro que incluyen el tallo cerebral y la corteza. Luego de cruzar la barrera hematoencefálica el virus se puede replicar en microglias generando un daño colateral a la barrera misma ${ }^{8}$. Es posible que tanto las fallas respiratorias como las neurales estén relacionadas con el daño del tronco encefálico, ya que el SARS-CoV-2 (al igual que el SARS-CoV) es capaz de entrar al cuerpo por el bulbo olfatorio mediante las fosas nasales e invadir el SNC, específicamente al tronco encefálico ${ }^{1,8}$.

\section{Material y métodos}

Esta investigación es una revisión sistemática, realizada bajo los criterios de la guía PRISMA (Preferred Reporting Items for Systematic Reviews and MetaAnalyses), usando como base de datos principal PubMed; como secundaria, se hizo uso de American Academy of Neurology (AAN); como base de datos terciaria se dispuso de SciELO; como cuaternaria fue ELSEVIER y, como de literatura gris se usó Medscape. Las formas de sesgo encontradas para la revisión están representadas en la herramienta de Robvis (Risk-Of-Bias VISualization). Fue utilizada al momento de iniciar la redacción, justo después de realizado el proceso de cribado y elegibilidad ya con los artículos seleccionados para tener constancia de la información relevante para la revisión (anexo 1).

A su vez, se utilizaron como herramienta de búsquedalos MeSH terms (MedicalSubject Headings): "COVID-19", "SARS-CoV-2", "manifestation", "neurology", "coronavirus"; y los Clinical Queries que sirvieron como un buscador más especializado gracias a los filtros metodológicos que tiene incorporados. Las variables evaluadas fueron: manifestaciones neurológicas asociadas a COVID-19, condiciones neurológicas preexistentes y la edad. Los criterios de inclusión fueron: (1) investigaciones científicas sobre COVID-19 publicadas durante el periodo 2019-2020, (2) investigaciones científicas redactadas en español y/o inglés, (3) investigaciones científicas sobre COVID-19 con enfoque en las manifestaciones neurológicas. Y los criterios de exclusión: (1) investigaciones enfocadas en otra cepa de los coronavirus diferentes al SARS-CoV-2, (2) artículos que no se encuentren en texto completo o estén terminados, (3) investigaciones que presenten la misma información y (4) aquellos trabajos que requieren de pago para acceder a la información del artículo.

Los resultados obtenidos se representaron en tablas y se dividieron entre revisión sistemática y estudios observacionales, ya que las revisiones muestran una metodología diferente al ser un conglomerado de investigaciones que ayudan beneficiosamente a dar validez a esta revisión, por lo que ciertos acápites no tienen que incluirse en otros artículos. 


\section{Resultados}
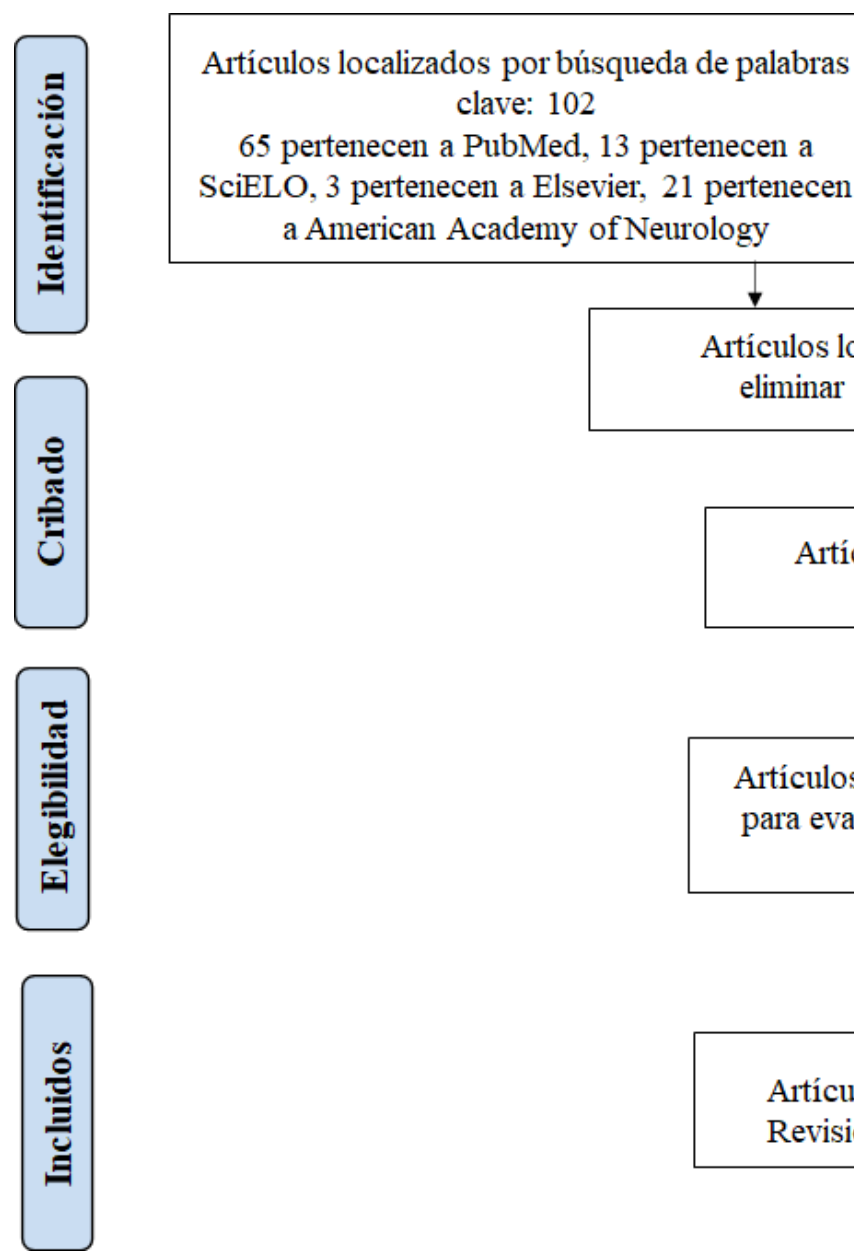

65 pertenecen a PubMed, 13 pertenecen a SciELO, 3 pertenecen a Elsevier, 21 pertenecen a American Academy of Neurology

Artículos localizados luego de eliminar duplicados: 123

Artículos localizados por búsqueda de palabras clave en literatura gris:

29

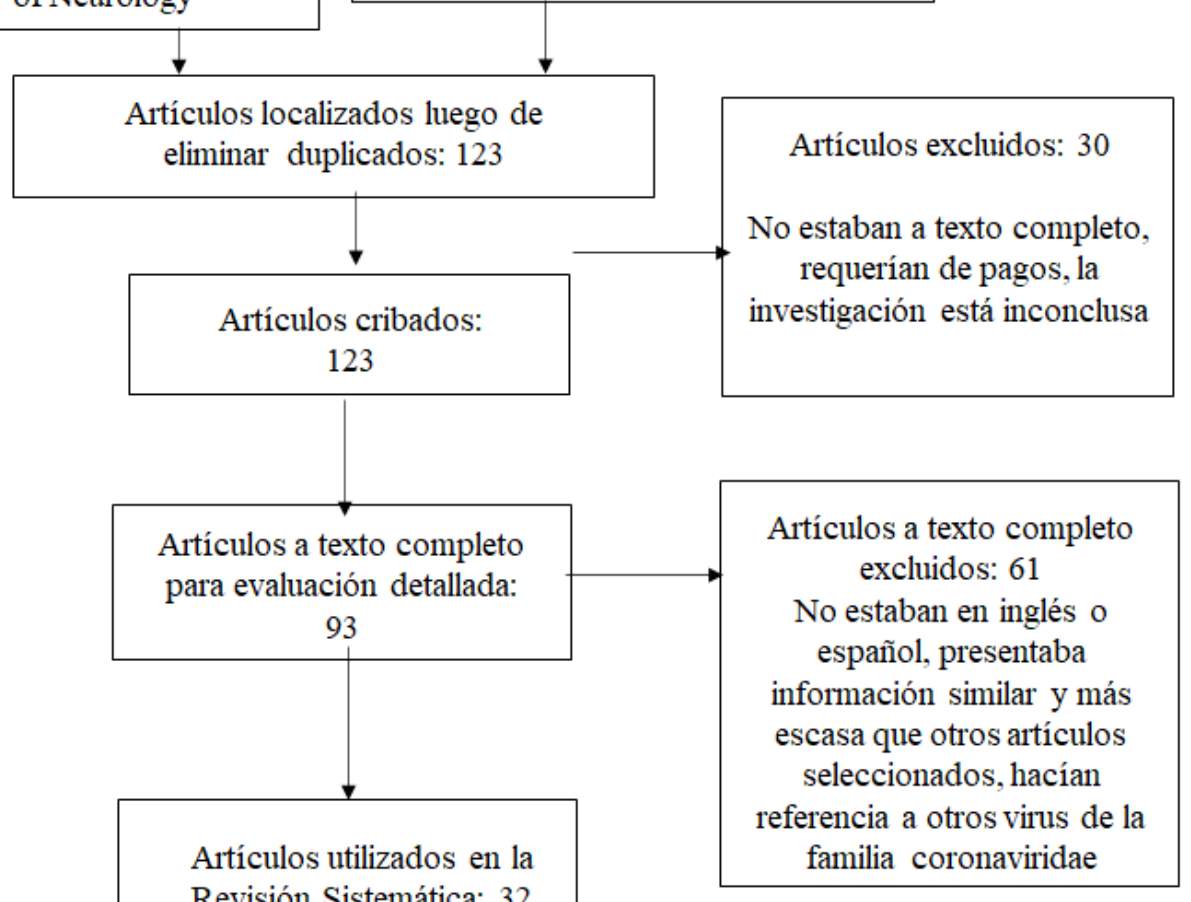

Figura 1. Diagrama de depuración de artículos

Fuente: Mena, Peńa, Polanco, Rosario, Ureña, Villegas, Yabra, Rojas, Reyes, Hernández, octubre 2020.

Por medio de este esquema se plantea el proceso de selección para la elaboración de la revisión sistemática. Durante el proceso de identificación de los artículos, el cual constó de cuatro fases: identificación de artículos relacionados, cribado de aquellos con criterios de exclusión, elegibilidad para los que cumplían los criterios de inclusión y la inclusión de los restantes. Se recolectaron un total de 131 artículos, que luego de un detallado proceso de elegibilidad y cribado, se redujo a 32 artículos en total para la elaboración de la revisión sistemática. 
Tabla 1. Manifestaciones neurológicas

\begin{tabular}{|c|c|c|}
\hline \multicolumn{3}{|c|}{ Estudios Observacionales } \\
\hline Estudio & Población (N) & Frecuencia de aparición (n) \\
\hline Ellul et al. (2020) & 125 & $\begin{array}{l}\text { Alteración del estado de conciencia (39), encefalopatía- (16), evento } \\
\text { cerebrovascular (ECV) (77) }\end{array}$ \\
\hline $\begin{array}{l}\text { Mendez-Guerrero } \\
\text { et al. }(2020)\end{array}$ & 1 & $\begin{array}{l}\text { Hiposmia (1), alteración de la conciencia (1), síndrome rígido- } \\
\text { acinético con anomalías oculares (1). }\end{array}$ \\
\hline $\begin{array}{l}\text { Pascual-Goño et } \\
\text { al. }(2020)\end{array}$ & 2 & $\begin{array}{l}\text { Diplopía (2), cefalea (2), hiposmia (1), parálisis del nervio abducen } \\
\text { derecho (2), parestesia (1), encefalopatía (2), alteración de la } \\
\text { conciencia (1), déficit de memoria episódica (2). }\end{array}$ \\
\hline $\begin{array}{l}\text { Madia et al. } \\
(2020)\end{array}$ & 6 & $\begin{array}{l}\text { Mialgia(4), síndrome de Guillain-Barré (SGB) (1), miopatía por } \\
\text { enfermedad crítica (CIM) o polineuropatía (2),cuadriplejía flácida } \\
\text { aguda (1). }\end{array}$ \\
\hline $\begin{array}{l}\text { Bodro et al. } \\
(2020)\end{array}$ & 2 & $\begin{array}{l}\text { Cefalea (2), parestesias del lado izquierdo y parestesia ipsilateral, } \\
\text { confusión y agitación (2), mialgias (2), alteración de la conciencia (1) }\end{array}$ \\
\hline Noro et al. (2020) & 1 & $\begin{array}{l}\text { Convulsiones (1) alteración de la conciencia (1), dificultad } \\
\text { para seguir las órdenes verbales (1) dificultad para movilizar las } \\
\text { extremidades (1) }\end{array}$ \\
\hline $\begin{array}{l}\text { Ashrafi et al. } \\
(2020)\end{array}$ & 6 & ECV (6), disartria (3), alteración de la conciencia (1) \\
\hline $\begin{array}{l}\text { Carod et al. } \\
(2020)\end{array}$ & 214 & Mareo (36), cefalea (28), hipogeusia (12), hiposmia (5) \\
\hline $\begin{array}{l}\text { Vergara et al. } \\
(2020)\end{array}$ & 1 & Cefalea (1), alteración de la consciencia (1) \\
\hline $\begin{array}{l}\text { McNamara et al. } \\
(2020)\end{array}$ & 2 & $\begin{array}{l}\text { Síndrome de Miller Fisher (1), diplopía (2), parestesias periorales } \\
\text { (1), mialgia (2), lumbalgia (1), anosmia (2), ageusia (2), polineuritis } \\
\text { craneal (1) }\end{array}$ \\
\hline $\begin{array}{l}\text { Batya et al. } \\
(2020)\end{array}$ & 214 & $\begin{array}{l}\text { Mareos (10), mialgia (45), alteración de la conciencia (3), ECV } \\
\text { (1), ataxia (1), convulsiones (7), ageusia (29), anosmia (34), visión } \\
\text { borrosa (1). }\end{array}$ \\
\hline $\begin{array}{l}\text { Hughes et al. } \\
(2020)\end{array}$ & 1 & Anosmia (1) \\
\hline Palao et al. (2020) & 1 & $\begin{array}{l}\text { Anosmia(1), ageusia(1), enfermedad desmielinizante del SNC(1), } \\
\text { esclerosis múltiple (1) }\end{array}$ \\
\hline $\begin{array}{l}\text { Anand et al. } \\
(2020)\end{array}$ & 7 & Mialgia (6), ageusia (2) \\
\hline Vaira et al. (2020) & 345 & Hiposmia (180), anosmia (61), hipogeusia (119), ageusia (36) \\
\hline $\begin{array}{l}\text { Carignan et al. } \\
(2020)\end{array}$ & 134 & Mialgia (10), anosmia (84), ageusia (84), visión borrosa (1) \\
\hline $\begin{array}{l}\text { García-Azorín et } \\
\text { al. }(2020)\end{array}$ & 576 & Esclerosis múltiple (EM) (3), cefalea (2), ataxia (6) \\
\hline
\end{tabular}




\begin{tabular}{|c|c|c|c|}
\hline \multicolumn{4}{|c|}{ Revisiones sistemáticas y metaanálisis } \\
\hline Estudio & $\begin{array}{l}\text { No. de } \\
\text { estudios } \\
\text { incluidos }\end{array}$ & $\begin{array}{l}\text { Población } \\
\text { (N) }\end{array}$ & Frecuencia de aparición (n) \\
\hline $\begin{array}{l}\text { Khatoon et al. } \\
(2020)\end{array}$ & 44 & 385 & $\begin{array}{l}\text { ECV (57), hemorragia intracerebral (9), alteración estado mental } \\
\text { (59), encefalopatías (19), ataxia (2), convulsiones (1), alteración de la } \\
\text { conciencia (2), Parálisis facial bilateral (2), SGB (2), parálisis flácida } \\
\text { aguda miembros inferiores (1), parestesia (1), disfagia (1), diplopía } \\
\text { (1), oscilopsia (1). }\end{array}$ \\
\hline $\begin{array}{l}\text { Ahmad et al. } \\
(2020)\end{array}$ & 17 & 14,543 & $\begin{array}{l}\text { Mareos (36), cefalea (5179), alteración de la conciencia (56), } \\
\text { hiposmia/ hipogeusia (4961), ECV (18), encefalopatía (150), mielitis } \\
\text { aguda (1), SGB (7), alteraciones musculares (23), epilepsia (2). }\end{array}$ \\
\hline $\begin{array}{l}\text { Niazkar et al. } \\
(2020)\end{array}$ & 6 & 480 & $\begin{array}{l}\text { Mareos (13), cefalea (9), hemorragia intracerebral (1), encefalopatías } \\
\text { (1). }\end{array}$ \\
\hline $\begin{array}{l}\text { Tsivgoulis et al. } \\
(2020)\end{array}$ & 16 & 1313 & $\begin{array}{l}\text { ECV (17), cefalea (150), mialgia (164), encefalopatías (2), trombosis } \\
\text { cerebral (1), alteración de la conciencia (66), SGB (5), hemorragia } \\
\text { intracerebral (1), parálisis del nervio facial (2). }\end{array}$ \\
\hline $\begin{array}{l}\text { Koralnik et al. } \\
(2020)\end{array}$ & 21 & 1,082 & $\begin{array}{l}\text { Mareos (36), Cefalea (30), ageusia/anosmia/hiposmia (408), ECV } \\
\text { (10), convulsiones (5), mielitis (1), encefalopatías (7), SGB (5), } \\
\text { alteración de la conciencia (57), otros (64). }\end{array}$ \\
\hline $\begin{array}{l}\text { Aghagoli et al. } \\
(2020)\end{array}$ & 7 & 574 & $\begin{array}{l}\text { Mareos (36), cefalea (41), alteración de la conciencia (56), encefalitis } \\
(2) \text {, meningitis (1), convulsiones (1). }\end{array}$ \\
\hline Pérez et al. (2020) & 3 & 44,813 & $\begin{array}{l}\text { Mialgia (8), náuseas (4), alteración de la conciencia (22), ataxia (2), } \\
\text { enfermedad cerebrovascular aguda (1) y convulsiones (78). }\end{array}$ \\
\hline $\begin{array}{l}\text { Vonck et al. } \\
(2020)\end{array}$ & 20 & 3498 & $\begin{array}{l}\text { ECV (305), ataxia (23), convulsión (549), ageusia/anosmia (1256), } \\
\text { alteración de la conciencia (278), hemorragia intracerebral ( } 7) \text {, } \\
\text { encefalopatías (187), arreflexia (4), parestesia (1) }\end{array}$ \\
\hline $\begin{array}{l}\text { Acharya et al. } \\
(2020)\end{array}$ & 3 & 3 & $\begin{array}{l}\text { Meningoencefalitis(1), ECV (1), SGB (1), fotofobia(1), anosmia/ } \\
\text { ageusia (1) }\end{array}$ \\
\hline $\begin{array}{l}\text { Egbert et al. } \\
(2020\end{array}$ & 26 & 361 & microhemorragias (16) \\
\hline Catal et al. (2020) & 32 & 143 & $\begin{array}{l}\text { Convulsiones (34), epilepsia (3), cefalea (68), alteración del estado } \\
\text { mental (10), parestesia (1), síndrome de encefalopatía hemorragia } \\
\text { posterior reversible (2), trombosis cerebral (14), encefalopatía } \\
\text { necrotizante hemorrágica aguda (1), encefalomielitis (1), meningitis/ } \\
\text { encefalitis (2) }\end{array}$ \\
\hline $\begin{array}{l}\text { Ghannam et al. } \\
(2020)\end{array}$ & 42 & 82 & Sx Miller Fisher (1), epilepsia (1), SGB (17), polineuritis craneal (1) \\
\hline $\begin{array}{l}\text { Wang et al. } \\
(2020)\end{array}$ & 41 & 3837 & $\begin{array}{l}\text { Fatiga }(1,274) \text {, mialgia (613), cefalea (352), anosmia (1370), ageusia } \\
(1266)\end{array}$ \\
\hline
\end{tabular}

Fuente: Mena et al., octubre 2020.

Por medio de artículos, se determinó en 17 estudios observacionales que las condiciones neurológicas que presentaron los pacientes diagnosticados con la COVID-19 son: ageusia, anosmia, cefalea, mialgia, alteración del estado de la conciencia, síndrome de Guillain-Barre y mareos. Por otro lado, en las 13 revisiones sistemáticas se hallaron, de igual manera, diversos casos de pacientes que presentaron 
el síndrome de Guillain-Barré, epilepsia, mareos, alteración de la conciencia, fatiga, meningitis y encefalitis. En menor frecuencia se observó también: trombosis cerebral, hemorragia intracerebral, ataxia, parestesia, mielitis aguda y lumbalgia.

Tabla 2. Condiciones neurológicas preexistentes

\begin{tabular}{|c|c|c|c|}
\hline \multicolumn{4}{|c|}{ Estudios Observacionales } \\
\hline Estudios & \multicolumn{2}{|c|}{ Población (N) } & Frecuencia de aparición (n) \\
\hline $\begin{array}{l}\text { Vergara et al, } \\
\quad(2020)\end{array}$ & \multicolumn{2}{|l|}{1} & Encefalomalacia (1) \\
\hline $\begin{array}{l}\text { Lennon et al. } \\
\quad(2020)\end{array}$ & \multicolumn{2}{|l|}{1} & Alzheimer (1) \\
\hline Anand et al. (2020) & \multicolumn{2}{|l|}{7} & $\begin{array}{l}\text { Enfermedad de Parkinson (1), Lesión cerebral traumática, derrame } \\
\text { cerebral (2) }\end{array}$ \\
\hline $\begin{array}{l}\text { García-Azorín et } \\
\text { al. (2020) }\end{array}$ & \multicolumn{2}{|l|}{576} & $\begin{array}{l}\text { ECV isquémico (35), hidrocefalia (4), polineuropatía (7), este- } \\
\text { nosis espinal (13) Neuro lupus (1), síndrome de malformación } \\
\text { (1), Alzheimer (28), enfermedad de Parkinson (11), enfermedad } \\
\text { de Neurobehçet (1), metástasis cerebral (1), glioblastoma multi- } \\
\text { forme (1), mielopatía (3), }\end{array}$ \\
\hline \multicolumn{4}{|c|}{ Revisiones sistemáticas y metaanálisis } \\
\hline Estudio & $\begin{array}{l}\text { No. de estudios } \\
\text { incluidos }\end{array}$ & $\begin{array}{l}\text { Población } \\
\text { (N) }\end{array}$ & Frecuencia de aparición (n) \\
\hline $\begin{array}{l}\text { Ahmad et al. } \\
\quad(2020)\end{array}$ & 17 & 14,543 & Enfermedad de Parkinson (1) \\
\hline $\begin{array}{l}\text { Koralnik et al. } \\
\quad(2020)\end{array}$ & 21 & 1,082 & Aneurisma cerebral (1) \\
\hline $\begin{array}{l}\text { Khatoon et al. } \\
\quad(2020)\end{array}$ & 44 & 385 & Enfermedad de Parkinson (1) \\
\hline $\begin{array}{l}\text { Niazkar et al. } \\
\quad(2020)\end{array}$ & 6 & 480 & Enfermedad de Parkinson (1) \\
\hline $\begin{array}{c}\text { Tsivgoulis et al. } \\
\text { (2020) }\end{array}$ & 16 & 1313 & Encefalomalacia temporal izquierda (1) \\
\hline $\begin{array}{l}\text { Ghannam et al. } \\
\text { (2020) }\end{array}$ & 42 & 82 & $\begin{array}{l}\text { Accidente cerebrovascular cardioembólico (3), CSVD (5), ECV } \\
\text { (27) }\end{array}$ \\
\hline $\begin{array}{l}\text { Ramin Zand et al. } \\
\text { (2020) }\end{array}$ & Metaanálisis & 17,799 & $\begin{array}{l}\text { Accidente cerebrovascular (156), accidente cerebrovascular isqué- } \\
\text { mico (123), hemorragia intracerebral (27), trombosis sinusal (6) }\end{array}$ \\
\hline
\end{tabular}

Fuente: Mena et al., octubre 2020.

Se determinó en cuatro estudios observacionales que las condiciones neurológicas preexistentes en los pacientes diagnósticos con la COVID-19 fueron encefalomalacia, Alzheimer, ECV isquémica, Alzheimer, Parkinson, polineuropatía, hidrocefalia, estenosis espinal, lesiones cerebrales traumáticas, mielopatía y cáncer. En las siete revisiones sistemáticas y/o metaanálisis se evidenció que condiciones preexistentes fueron: ECV isquémico y hemorrágicos, Parkinson, trombosis sinusal, encefalomalacia y aneurisma cerebral. 
Tabla 3. Rango de edades

\begin{tabular}{|c|c|c|c|}
\hline \multicolumn{4}{|c|}{ Estudios Observacionales } \\
\hline Estudio & \multicolumn{2}{|l|}{ Población (N) } & Frecuencia de aparición (n) \\
\hline Méndez-Guerrero et al. (2020) & \multicolumn{2}{|l|}{1} & $>60$ a $(1)$ \\
\hline Pascual-Goño et al. (2020) & \multicolumn{2}{|l|}{2} & $>60 \mathrm{a}(1),>30 \mathrm{a}(1)$ \\
\hline Madia et al. (2020) & \multicolumn{2}{|l|}{6} & $>50$ a (6) \\
\hline Noro et al. (2020) & \multicolumn{2}{|l|}{1} & $>60 \mathrm{a}(1)$ \\
\hline Ashrafi et al. (2020) & \multicolumn{2}{|l|}{6} & $>55 \mathrm{a}(6)$ \\
\hline Vergara et al. (2020) & \multicolumn{2}{|l|}{1} & $>70$ a $(1)$ \\
\hline McNamara et al. (2020) & \multicolumn{2}{|l|}{2} & $<50$ a $(2)$ \\
\hline Anand et al. (2020) & \multicolumn{2}{|l|}{7} & $<50 \mathrm{a}(2),>65 \mathrm{a}(5)$ \\
\hline Vaira et al. (2020) & \multicolumn{2}{|l|}{345} & $<50$ a (146), > 65 a (199) \\
\hline Carignan et al. (2020) & \multicolumn{2}{|l|}{134} & $<50$ a $(134)$ \\
\hline García-Azorín et al. (2020) & \multicolumn{2}{|l|}{76} & $>50$ a (249), < 50 a (327) \\
\hline Bodro et al. (2020) & \multicolumn{2}{|l|}{2} & $>40 \mathrm{a}(1),>20 \mathrm{a}(1)$ \\
\hline \multicolumn{4}{|c|}{ Revisiones sistemáticas y metaanálisis } \\
\hline Estudio & No. de estudios incluidos & $\begin{array}{l}\text { Pobla- } \\
\text { ción }(\mathrm{N})\end{array}$ & Frecuencia de aparición (n) \\
\hline Ahmad et al (2020) & 17 & 14,543 & $>50$ a $(392),<50$ a $(1)$ \\
\hline Koralkik et al (2020) & 21 & 1,082 & $>50 \mathrm{a}(93),<50 \mathrm{a}(33)$ \\
\hline
\end{tabular}

Fuente: Mena et al., octubre 2020.

En la revisión realizada se observó que en 12 artículos de estudios observacionales el grupo etario más frecuente a presentar manifestaciones neurológicas en personas diagnosticadas con COVID-19 están alrededor de los 50 hasta los 70 ańos, en menor medida se vio también que el grupo etario menos frecuente y por lo tanto con menor riesgo de presentar manifestaciones neurológicas rondaba entre los 20 y 30 años. De igual manera en dos estudios de revisiones sistemáticas se observó que el grupo etario común se encontraba entre los 50 y 60 años.

\section{Discusión y conclusiones}

\section{Resumen de evidencias}

Los datos recolectados en esta revisión estudiaron tres variables que pueden influir en la presentación de manifestaciones neurológicas en pacientes diagnosticados con COVID-19. Estas fueron las condiciones neurológicas preexistentes, las manifestaciones neurológicas provocadas por el virus, y el rango de edad de los pacientes. 
Las condiciones neurológicas preexistentes más frecuentes fueron: el Parkinson, eventos cerebrovasculares y Alzheimer (véase tabla 1). Como manifestaciones neurológicas más comunes se observaron: hiposmia, ageusia, cefalea, mialgia, alteración de la conciencia y convulsiones (véase tabla 2); y el rango de edad observado que presentó manifestaciones neurológicas va desde los 45 a los 80 años (véase tabla 3).

Al comparar los resultados entre los estudios utilizados, se pudo identificar cierto grado de concordancia en lo que a manifestaciones neurológicas y frecuencia de aparición se refiere. El estudio realizado por los neurólogos Jean Paul Vergara y Carlos Tolosa encontró que la frecuencia de aparición de los síntomas neurológicos era: mareos (17\%), cefalea (13\%), hipogeusia (6\%) e hiposmia (5\%). Se evidenció, además, que la enfermedad cerebrovascular aguda tuvo un porcentaje de aparición de $6 \%$, así como la alteración de la conciencia con un $15 \%$ y el compromiso musculoesquelético subsecuente de un $19 \%{ }^{3}$. Evidenciando en sus conclusiones que la relación entre el virus y la presentación de manifestaciones neurológicas en los pacientes es frecuente.

De la misma manera, el primer estudio de cohorte, acerca de las manifestaciones neurológicas de la COVID-19, resumió que presentaron 78 de 214 pacientes $(36.4 \%)$ hospitalizados en tres hospitales designados para COVID-19 en China. Destacando que 6 presentaron derrames cerebrales, pero que los síntomas neurológicos más comunes en los pacientes fueron: hiposmia, mareos, cefalea, mialgia, hipogeusia y disgeusia ${ }^{19}$. Reportando así que de estos los síntomas más frecuentes fueron mialgia y cefaleas presentándose en un $52 \%$ de los pacientes, al igual que la hiposmia e hipogeusia ${ }^{19}$. Observando así similitud entre los resultados de diversos estudios y la revisión en sí.

\section{Limitaciones del estudio}

Entre las limitaciones de esta revisión se encuentra la heterogeneidad de los estudios debido a que es un tema que se está investigando exponencialmente; por lo que se debe tener presente al momento de elegir artículos ya que se puede incluir algunos que no provean la información correspondiente en su totalidad. Otra limitación fue que la mayoría de los trabajos que hablaban en sí de las manifestaciones neurológicas se realizaron con uno o dos pacientes, lo cual limitó la utilización de casos variados para la revisión. Dentro de otras limitaciones, al momento de tabular la variable del sexo en la revisión, fue que tampoco se especificaba en la mayoría de los artículos ya que se refería a los sujetos observados como pacientes en general, muy pocos artículos especificaban ambos sexos (masculino y femenino), por lo cual no se tomaron en cuenta al momento de analizar esta variable en los resultados de esta revisión. Por último, otra de las limitaciones presentadas en esta investigación se encuentra la variabilidad entre estudios o los errores en la fase de análisis, producto de la publicación duplicada de estos o la omisión de trabajos publicados en un idioma distinto al inglés o español.

\section{Principales conclusiones}

Las manifestaciones neurológicas que se pueden presentar durante una infección por COVID-19 son varias, y como se pudo observar en esta revisión bibliográfica las mismas son frecuentes en los pacientes durante el curso de su enfermedad y con un grado variable de severidad. Al este virus comprometer a otros sistemas y tener manifestaciones marcadas a nivel respiratorio y circulatorio, se ha limitado la cantidad de investigaciones sobre las afecciones al sistema nervioso. Sin embargo, hoy en día las nuevas evidencias apuntan a que existe una relación entre el sistema nervioso y la enfermedad causada por SARS-Cov 2. Aun así, se deben realizar más estudios clínicos, para entender correctamente cómo se afecta el sistema nervioso en su totalidad. 
Anexo 1. Resumen del riesgo de sesgo en los artículos individuales
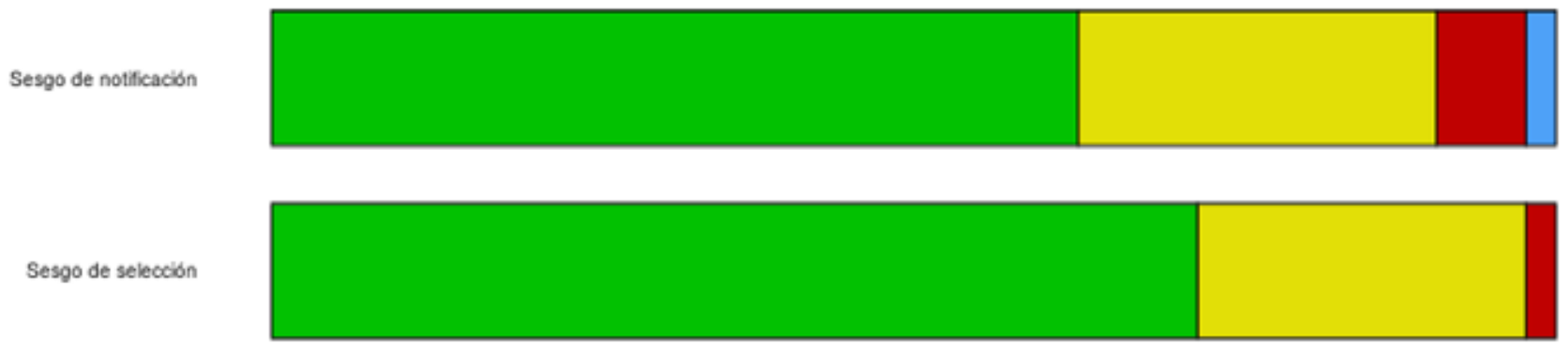

Variable de contusión
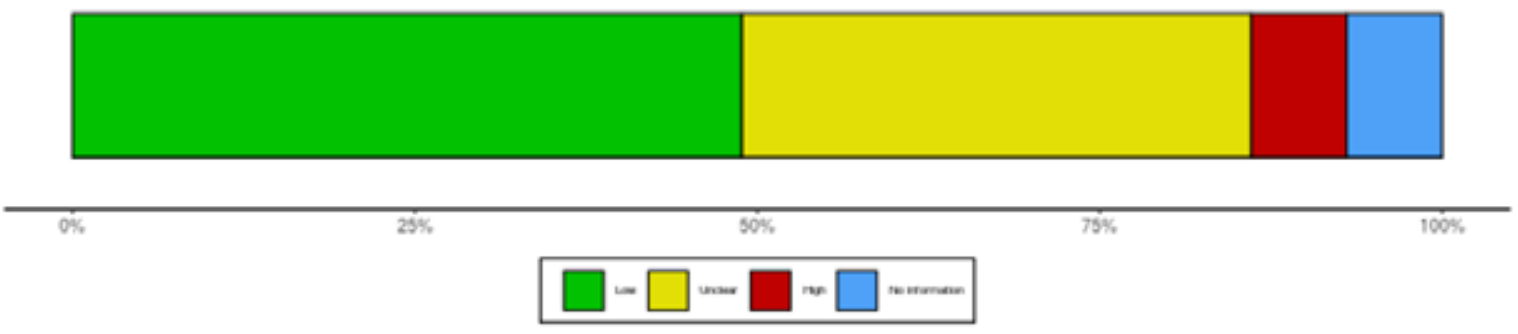

Fuente: Mena et al., octubre 2020.

\section{Bibliografía}

1. Wang L, Shen Y, Li M, Chuang H, Ye Y, Zhao $\mathrm{H}$, et al. Clinical manifestations and evidence of neurological involvement in 2019 novel coronavirus SARS-CoV-2: a systematic review and meta-analysis. J Neurol. 2020;267(10):2777-89.

2. Niazkar H, Zibaee B, Nasimi A, Bahri N. The neurological manifestations of COVID-19: a review article. Neurol Sci. 2020;41(7):1667-71.

3. Vergara J, Tolosa C. Covid 19: manifestaciones neurológicas. Acta neurol colomb. 2020;36 (2):7-10.

4. Ahmad I, Rathore F. Neurological manifestations and complications of COVID-19: A literature review. J Clin Neurosci. 2020;77:8-12.

5. Koralnik I, Tyler K. COVID-19: A global threat to the nervous system. Ann Neurol. 2020;88(1):1-11.

6. Khatoon F, Prasad K, Kumar V. Neurological manifestations of COVID-19: available evidences and a new paradigm. J Neurovirol. 2020;26(5):619-30.

7. Carignan A, ValiquetteL, Grenier C, MusoneraJB, Nkengurutse D, Marcil-Héguy A, et al. Anosmia and dysgeusia associated with SARS-CoV-2 infection: an age-matched case-control study. CMAJ. 2020;192(26):702-7.

8. Acharya A, Kevadiya BD, Gendelman HE, Byrareddy SN. SARS-CoV-2 infection leads to neurological dysfunction. J Neuroimmune Pharmacol. 2020;15(2):167-73.

9. Ellul MA, Benjamin L, Singh B, Lant S, Michael BD, Easton A, et al. Neurological associations of COVID-19. Lancet Neurol. 2020;19(9):767-83.

10. Méndez-Guerrero A, Laespada-García MI, Gómez-Grande A, Ruiz-Ortiz M, Blanco-Palmero VA, Azcarate-Diaz FJ, et al. Acute hypokinetic-rigid syndrome following SARS-CoV-2 infection. Neurology. 2020;95(15):2109-e2118. 
11. Pascual-Gońi E, Fortea J, Martínez-Domeño A, Rabella N, Tecame M, Gómez-Oliva C, et al. COVID-19-associated ophthalmoparesis and hypothalamic involvement. Neurol Neuroimmunol Neuroinflamm. 2020;7(5):e823.

12. Madia F, Merico B, Primiano G, Cutuli SL, De Pascale G, Servidei S. Acute myopathic quadriplegia in patients with COVID-19 in the intensive care unit. Neurology. 2020;95(11):492-4.

13. Bodro M, Compta Y, Llansó L, Esteller D, Doncel-Moriano A, Mesa A, et al. Increased CSF levels of IL-1 $\beta$, IL-6, and ACE in SARSCoV-2-associated encephalitis. NeurolNeuroimmunol Neuroinflamm. 2020;7(5):e821.

14. Noro F, de Mendonça Cardoso F, Marchiori E. COVID-19 and posterior reversible encephalopathy syndrome. Neurol Clin Pract. 2021;11 (2):e202-4.

15. Ashrafi F, Zali A, Ommi D, Salari M, Fatemi A, Arab-Ahmadi M, et al. COVID-19-related strokes in adults below 55 years of age: a case series. Neurol Sci. 2020;41(8):1985-9.

16. Carod-Artal FJ. Complicaciones neurológicas por coronavirus y COVID-19. Rev Neurol. 2020;70(9):311-22.

17. McNamara D. Two rare neurologic conditions linked to COVID-19 [Internet]. Medscape. 2020. Disponible en: https://www.medscape. com/viewarticle/929631

18. Yasgur BS, MA, LSW. COVID-19: First data confirm neurologic symptoms common [Internet]. Medscape. 2020. Disponible en: https://www. medscape.com/viewarticle/928848

19. Hughes S. COVID-19 neurological effects: Does virus directly attack the brain? [Internet]. Medscape. 2020. Disponible en: https://www. medscape.com/viewarticle/931658
20. Palao M, Fernández-Díaz E, Gracia-Gil J, Romero-SánchezCM, Díaz-MarotoI,SeguraT.Multiple sclerosis following SARS-CoV-2 infection. Mult Scler Relat Disord. 2020;45(102377):102377.

21. Anand P, Al-Faraj A, Sader E, Dashkoff J, Abdennadher M, Murugesan R, et al. Seizure as the presenting symptom of COVID-19: A retrospective case series. Epilepsy Behav. 2020; 112:107335.

22. Vaira LA, Hopkins C, Salzano G, Petrocelli M, Melis A, Cucurullo M, et al. Olfactory and gustatory function impairment in COVID-19 patients: Italian objective multicenter-study. Head Neck. 2020;42(7):1560-9.

23. García-Azorín D, Martínez-Pías E, Trigo J, Hernández-Pérez I, Valle-Peñacoba G, Talavera $\mathrm{B}$, et al. Neurological comorbidity is a predictor of death in covid-19 disease: A cohort study on 576 patients. Front Neurol. 2020;11:781.

24. Tsivgoulis G, Palaiodimou L, Katsanos AH, Caso V, Köhrmann M, Molina C, et al. Neurological manifestations and implications of COVID-19 pandemic. Ther Adv Neurol Disord. 2020;13:1756286420932036.

25. Aghagoli G, Gallo Marin B, Katchur NJ, Chaves-Sell F, Asaad WF, Murphy SA. Neurological involvement in COVID-19 and potential mechanisms: A review. Neurocrit Care. 2021;34(3):1062-71.

26. Pérez CA. Looking ahead: The risk of neurologic complications due to COVID-19. Neurol Clin Pract. 2020;10(4):371-4.

27. Vonck K, Garrez I, De Herdt V, Hemelsoet D, Laureys G, Raedt R, et al. Neurological manifestations and neuro-invasive mechanisms of the severe acute respiratory syndrome coronavirus type 2. Eur J Neurol. 2020;27(8):1578-87. 
28. Egbert AR, Cankurtaran S, Karpiak S. Brain abnormalities in COVID-19 acute/subacute phase: A rapid systematic review. Brain Behav Immun. 2020;89:543-54.

29. Katal S, Balakrishnan S, Gholamrezanezhad A. Neuroimaging and neurologic findings in COVID-19 and other coronavirus infections: A systematic review in 116 patients. J Neuroradiol. 2021;48(1):43-50.

30. Ghannam M, Alshaer Q, Al-Chalabi M, Zakarna L, Robertson J, Manousakis G. Neurological involvement of coronavirus disease 2019: a systematic review. J Neurol. 2020;267(11):3135-53.
31. Lennon JC. Neurologic and immunologic complications of COVID-19: Potential longterm risk factors for Alzheimer's disease. J Alzheimers Dis Rep. 2020;4(1):217-21.

32. Shahjouei S, Naderi S, Li J, Khan A, Chaudhary D, Farahmand G, et al. Risk of stroke in hospitalized SARS-CoV-2 infected patients: A multinational study. EBioMedicine. 2020;59:102939. 